\title{
X-ray properties of G308.3-1.4 and its central compact object
}

\author{
K. A. Seo ${ }^{1}$, C. Y. Hui ${ }^{1}$, R. H. H. Huang ${ }^{2}$, L. Trepl ${ }^{3}$, T.-N. Lu ${ }^{2}$, \\ A. K. H. Kong ${ }^{2} \dagger$ and F. M. Walter ${ }^{4}$ \\ ${ }^{1}$ Department of Astronomy and Space Science, Chungnam National University, \\ Daejeon 305-764, Korea \\ ${ }^{2}$ Institute of Astronomy and Department of Physics, National Tsing Hua University, \\ Hsinchu, Taiwan \\ ${ }^{3}$ Astrophysikalisches Institut und Universitäts-Sternwarte, Universität Jena, \\ Schillergäßchen 2-3, 07745 Jena, Germany \\ ${ }^{4}$ Department of Physics and Astronomy, Stony Brook University, Stony Brook, NY \\ 11794-3800, USA
}

\begin{abstract}
We present a short Chandra observation that confirms a previous unidentified extended X-ray source, G308.3-1.4, as a new supernova remnant (SNR) in the Milky Way. Apart from identifying its SNR nature, a bright X-ray point source has also been discovered at the geometrical center. Its X-ray spectral properties are similar to those of a particular class of neutron star known as central compact objects (CCOs). On the other hand, the optical properties of this counterpart suggests it to be a late-type star. Together with the interesting $\sim 1.4$ hours $\mathrm{X}$-ray periodicity found by Chandra, this system can possibly provide the first direct evidence of a compact binary survived in a supernova explosion.
\end{abstract}

Keywords. supernova remnants, X-rays

\section{Introduction}

Recently, we initiated an extensive identification campaign of unidentified extended ROSAT All-Sky Survey (RASS) objects (Hui et al. 2012). The brightest target in our campaign, G308.3-1.4, has already been known as a SNR candidate in the MOST SNR catalogue (Whiteoak 1992). But the limited photon statistics and the poor resolution of the RASS data do not allow any further probe of its X-ray emission properties. This has motivated us to observe G308.3-1.4 with the Chandra X-ray Observatory. The analysis of this observation is detailed in Hui et al. (2012); in these proceedings, we present a highlight of the major results.

\section{Confirmation of G308.3-1.4 as a new SNR}

The X-ray image of the field around G308.3-1.4 obtained by Chandra is displayed in Figure 1. An incomplete shell-like X-ray structure is found to be well-correlated with the radio shell structure. The radio contours are obtained from the $843 \mathrm{MHz}$ Sydney University Molonglo Sky Survey (Bock et al. 1999). Together with the X-ray spectral analysis of the extended emission which suggests it is a shock-heated plasma with a temperature in a range of $k T \sim 0.6-1 \mathrm{keV}$ (see Table 2 and Fig. 8 in Hui et al. 2012), our observation unambiguously confirms G308.3-1.4 as a new SNR. A recent radio

\section{$\dagger$ Golden Jade Fellow of Kenda Foundation, Taiwan}




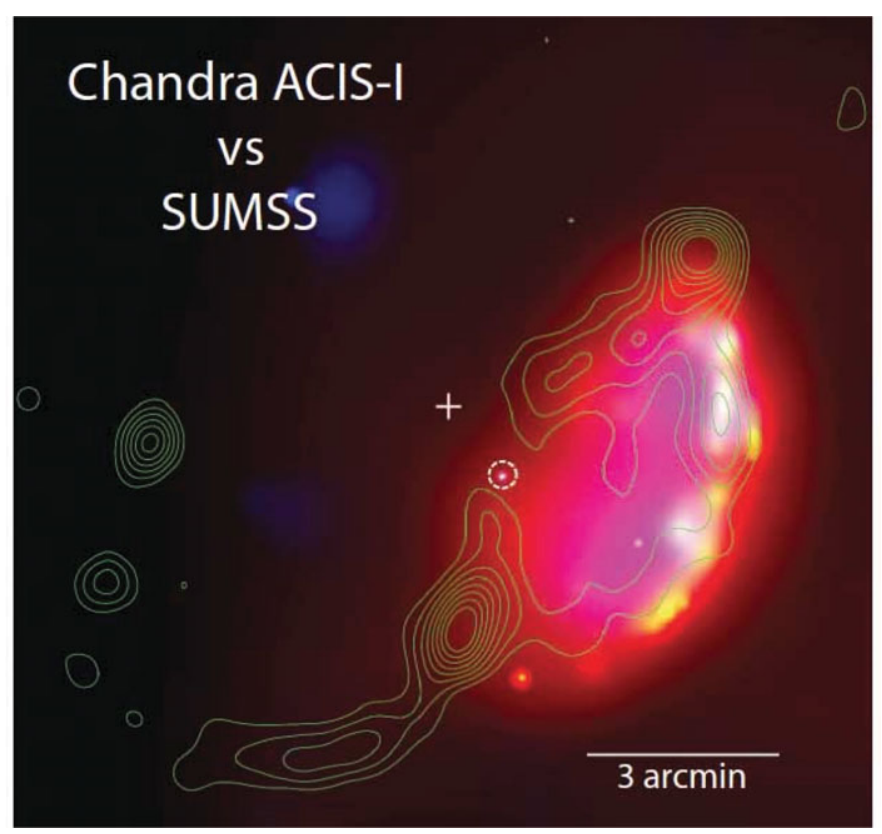

Figure 1. $10^{\prime} \times 10^{\prime}$ Chandra ACIS-I X-ray colour image of G308.3-1.4 (red: 0.5 - $1 \mathrm{keV}$, green: $1-2 \mathrm{keV}$, blue: $2-8 \mathrm{keV}$ ). The binning factor of this image is 2 . Adaptive smoothing has been applied to achieve a minimum signal-to-noise ratio of 3 . The geometrical center inferred from the X-ray morphology is illustrated by the cross. A bright source which locates closest to the geometrical center is highlighted by the dash circle. Top is north and left is east.

investigation has come the same conclusion, suggesting G308.3-1.4 is a young to middleaged SNR in the early adiabatic phase of evolution (De Horta et al. 2012).

\section{Discovery of a new central compact object associated with G308.3-1.4}

Apart from confirming the SNR nature of G308.3-1.4, our Chandra observation also enables us to search for the possible stellar remnant formed in the supernova explosion. Among 17 newly detected X-ray point sources (cf. Table 1 in Hui et al. 2012), the brightest source is the one located closest to the geometrical center of G308.3-1.4 (see Fig. 1). Its X-ray point source spectrum can be described by a double blackbody with the temperature of $k T_{1} \simeq 0.1 \mathrm{keV}, k T_{2} \simeq 0.4 \mathrm{keV}$ and emitting areas of $R_{1} \simeq 27 D_{\mathrm{kpc}} \mathrm{km}$ and $R_{2} \simeq 35 D_{\mathrm{kpc}} \mathrm{m}$ respectively (see Fig. 2 ), where $D_{\mathrm{kpc}}$ is the distance to G308.31.4 in units of $1 \mathrm{kpc}$. These are similar to those of CCOs - one of the most enigmatic manifestations of neutron stars (cf. Hui et al. 2006, 2009, 2012). The column density inferred from the CCO spectrum is consistent with that for the remnant, which suggest the possible association between the CCO and G308.3-1.4. We proceeded to search for the possible X-ray periodic signals from $\mathrm{CCO}$ and have found an interesting periodicity candidate of $P \sim 1.4 \mathrm{hrs}$ (Fig. 3). Together with the spectral energy distribution of its identified optical/IR counterpart, which conforms with the spectrum of a M dwarf, our results suggest a possible direct evidence for compact binary that survived in a supernova explosion (Hui et al. 2012). 


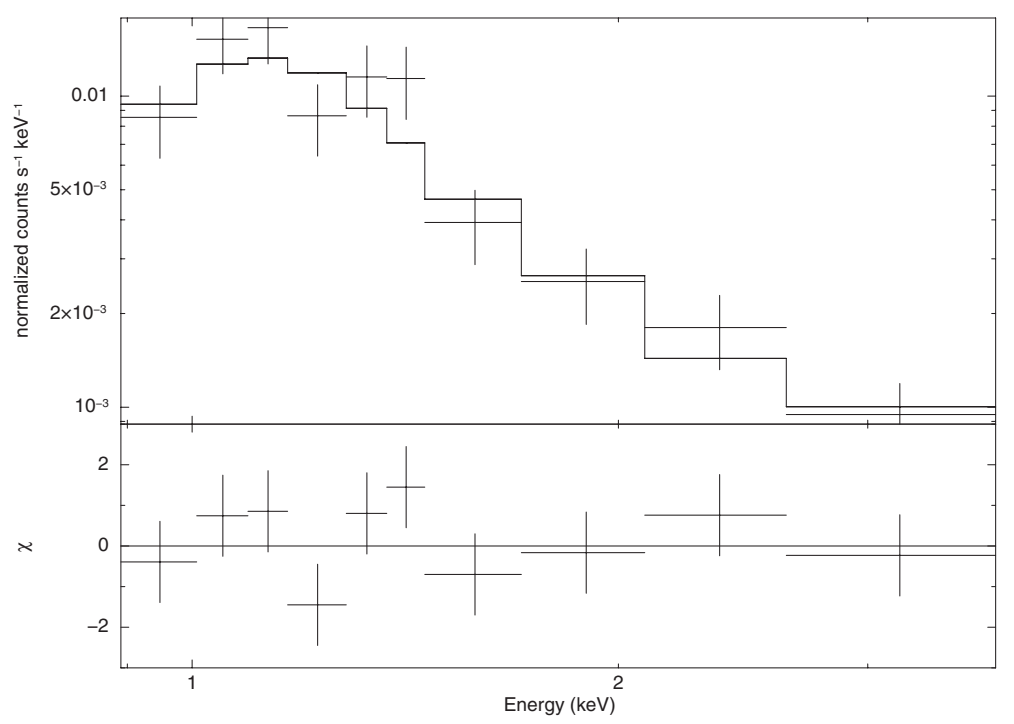

Figure 2. X-ray spectrum of the emission from the position of CCO as observed with ACIS-I with the best-fit double blackbody model (upper panel) and contributions to the $\chi^{2}$ statistics (lower panel). The error bars represent $1 \sigma$ uncertainties.

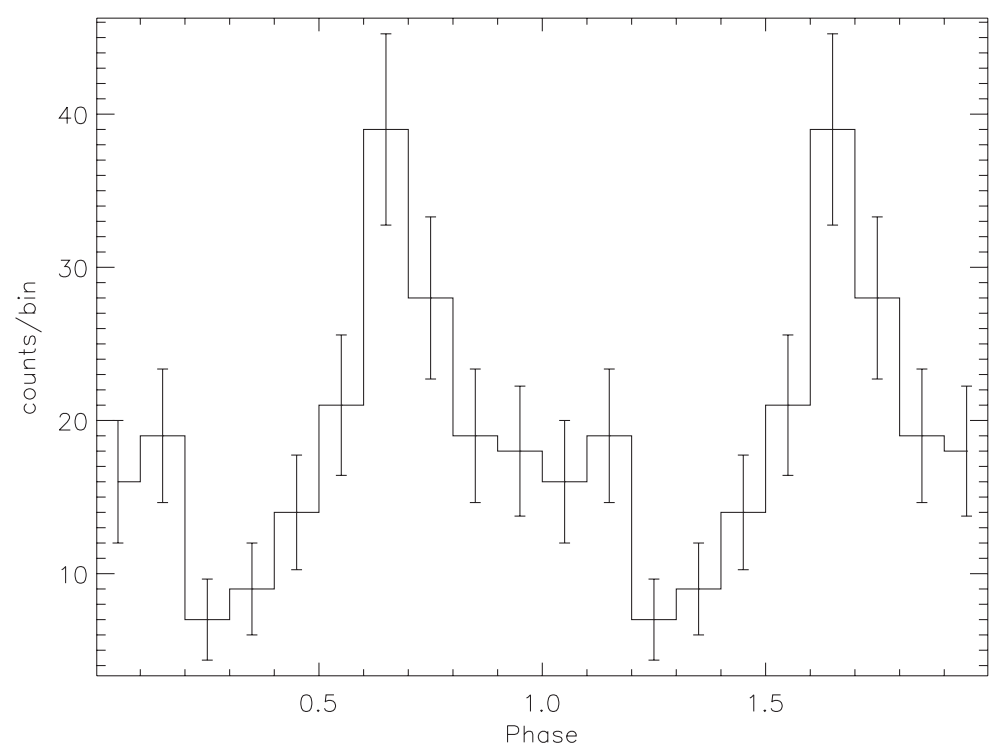

Figure 3. X-ray counts of CCO versus phase for a periodicity candidate of $1.4 \mathrm{hrs}$. Two periodic cycles are shown for clarity. The error bars represent $1 \sigma$ uncertainties.

\section{References}

Bock, D., M. I. Large, M. I., \& Sadler, E. M. 1999, AJ, 117, 1578

De Horta, A. Y., et al. 2012, arXiv:1210.2781v1

Hui, C. Y., et al. 2012, ApJ, 750, 7

Hui, C. Y. \& Becker, W. 2009, A\&BA, 494, 1005

Hui, C. Y. \& Becker, W. 2006, A\&A, 454, 543

Whiteoak, J. B. Z. 1992 A $\& A, 262,251$ 